\title{
Letter to the Editor: A response to Horne and Lucey (2017)
}

\author{
John A. Carver, ${ }^{*}$ David C. Thorn, ${ }^{*}$ Heath Ecroyd, $\dagger$ and Carl Holt ${ }^{1}$ \\ ${ }^{*}$ Research School of Chemistry, College of Physical and Mathematical Sciences, The Australian National University, Acton, \\ Australian Capital Territory 2601, Australia \\ †School of Biological Sciences and Illawarra Health and Medical Research Institute, University of Wollongong, Wollongong, \\ New South Wales 2522, Australia \\ łInstitute of Molecular, Cellular and Systems Biology, University of Glasgow, Glasgow, G12 8QQ, United Kingdom
}

We wrote a review in the Journal of Dairy Science in 2013 (Holt et al., 2013) and another in the International Dairy Journal in 2015 (Thorn et al., 2015), both dealing with the application of modern protein science concepts to the chemistry and physics of caseins. These were substantial documents with an important message. We brought together results from the burgeoning new fields of intrinsically disordered proteins (IDP), amyloid fibril formation, and molecular chaperone action to provide a radically new view of caseins and the casein micelle. We showed, through various examples, that caseins were far from unique among proteins in their behavior and that other IDPs provided better models for understanding caseins than either globular proteins or soap molecules. New concepts have been developed within the IDP field to explain how IDPs interact with each other and with intermediately folded globular proteins to form amyloid fibrils, amorphous aggregates, gels, or precipitates. These ideas relate to interactions between main-chain groups having low sequence specificity (also called promiscuous interactions) that are important in the action of caseins as molecular chaperones and in forming amorphous aggregates such as the casein micelle. Highly sequence-specific interactions, by contrast, can lead to the cross- $\beta$-sheet core structure of amyloid fibrils. Work on IDPs by Rohit Pappu and his colleagues has revealed that some IDPs contain polar sequences called polar tracts (van der Lee et al., 2014) that can form tertiary or quaternary structures in spite of their hydrophilic character. We identified either 1 or 2 polar tract-type sequences in all caseins, each generally rich in Pro (P) and Gln $(\mathrm{Q})$ residues, and proposed that casein $\mathrm{P}, \mathrm{Q}-$-rich sequences interact promiscuously to give rise to open and highly hydrated amorphous structures, including casein micelles.

Our reviews showed how exciting new ways of thinking about caseins and casein micelles are possible. They have proved popular with many casein researchers, as

Received April 20, 2017.

Accepted April 24, 2017.

${ }^{1}$ Corresponding author: carl.holt@glasgow.ac.uk was recognized in 2016 when we were awarded the Journal of Dairy Science Most-Cited Paper Award (Dairy Foods) by the American Dairy Science Association for the 2013 review article (Holt et al., 2013). In the process of developing our argument, some long-established notions about the nature of caseins were re-evaluated, one of which was the relative importance of the hydrophobic effect in casein-casein interactions. In proteins, the hydrophobic interaction is defined to be an interaction of side chains (Kauzmann, 1959). It is well known through numerous examples (Uversky et al., 2000; van der Lee et al., 2014) that IDPs, including caseins, are less hydrophobic than the vast majority of globular proteins. Indeed, this must be so because otherwise the IDPs would tend to adopt a compact conformation from which water molecules are largely excluded. The interactions of IDPs are qualitatively the same as in globular proteins but the balance is different in most cases, with less importance given to the hydrophobic effect between side chains and more importance given to interactions of the main chain. In general, both sidechain and main-chain interactions involve a combination of hydrogen bonding interactions and desolvation or bridging by water molecules, together with other types of interaction. Both types may be endothermic and both can be broken or reduced by detergents or high concentrations of chaotropic agents such as urea. Distinguishing between them may be difficult.

Our views have attracted critical commentary from Horne and Lucey (Horne, 2017; Horne and Lucey, 2017) because of their growing acceptance and potential impact on the reasonableness of the dual-binding model of casein micelles, first proposed by Horne (1998). The rationale of the dual-binding model is that caseins are analogous to giant soap molecules with charged hydrophilic heads and long hydrophobic tails. In the first sentence of the abstract of his 1998 paper proposing the model, Horne wrote "This paper reviews the literature on the interactions of the caseins and suggests that the state of association of these proteins is governed by a balance of attractive hydrophobic interactions and electrostatic repulsion." The role of calcium phosphate in 
the balance of forces is envisaged to be through the neutralization of the net negative charge. In a later review (Horne, 2006), Horne explained his unique concept of hydrophobic attraction through the following sentence. "Horne (Horne, 2002) grouped such interactions under the label hydrophobic but this should be considered a general term encompassing true hydrophobic attraction as well as hydrogen bonds." Combining hydrophobic interactions and hydrogen bonding into one attractive principle and then continuing to call it a hydrophobic attraction cannot be justified. These are 2 distinct effects, arising from different physical principles, operating in the main on different parts of the polypeptide chain - the hydrophobic effect of the interacting side chains and the hydrogen bonds formed between main chains.

According to the well-established principles of thermodynamics, the equilibrium size of a micelle is properly expressed by the achievement of a minimum in the free energy of a system containing the micelles and their constituent ions and protein, rather than through Horne's proposed balancing of forces. Thus, we can express the total free energy of forming casein micelles as a sum of free energy terms including the change in electrostatic free energy (combining attractive and repulsive interactions), the free energy of forming the calcium phosphate nanoclusters and their sequestration by caseins, hydrogen bonding changes (mainly associated with the backbone), and the hydrophobic effect of side chains, resulting in desolvation of parts of the interacting casein molecules, the change in conformational free energy, and other terms such as bridging interactions in the formation of cysteine or salt bridges. The magnitudes of the terms in the summation are largely unknown. In the dual-binding model, only 2 terms are present in the summation, the hydrophobic free energy and the electrostatic repulsion of the head groups because of the supposed close similarity of caseins to giant soap molecules. In an alternative nanocluster model (De Kruif and Holt, 2003), no such simplifications were made: they stated that "the integrity of micelles depends on strong linkages to calcium phosphate, together with some less clearly defined factors such as hydrogen bonding, salt bridging, and entropic driving forces such as chain entanglements and desolvation."

We too think that the analogy with a soap molecule is simplistic and that the likely importance of the hydrophobic effect has been overestimated in the past. The overestimation arose in part through the widespread use of the Bigelow scale of residue hydrophobicity (Bigelow, 1967) to measure the average hydrophobicity of caseins. Using this scale, Bigelow found that $\alpha$-casein was more hydrophobic than $88.5 \%$ of his sample of more than 150 other, mainly globular, proteins. The Bigelow scale is now mostly forgotten outside the casein field for 3 reasons. First, the scale has no experimental hydrophobicity values for the side chains of 5 amino acids and Bigelow's assumed values were subsequently shown to be incorrect. Second, the scale has a high hydrophobicity for Pro, classifying it as one of the most hydrophobic residues, even though Pro is seldom buried inside globular proteins (Janin, 1979; MacArthur and Thornton, 1991). Third, other hydrophobicity scales have since been developed with values for all the amino and imino acids, and Pro is now generally classified as a polar or even hydrophilic imino acid. Moreover, it has been found to have the highest propensity of any amino acid residue to be found in the solvent-exposed, disordered regions of proteins (Huang et al., 2014). We have repeated the hydrophobicity calculations on caseins using the scale of Kyte and Doolittle (1982). The use of this scale rather than any other is easy to justify because it is widely used in current protein science and, in conjunction with either the fractions of positively and negatively charged residues or normalized net charge, is used quite generally, and with a high success rate, to discriminate between folded proteins and IDPs (Uversky et al., 2000; Mao et al., 2010; Huang et al., 2014). Using the Kyte and Doolittle scale or an optimized IDP hydropathy scale (Huang et al., 2014), caseins are predicted correctly to be IDPs and to be more hydrophilic, on average, than the great majority of globular proteins (Redwan et al., 2015). Using the Bigelow scale, caseins, especially $\beta$-casein, are predicted to be among the most hydrophobic proteins known to science and to be globular. Thus, according to current practice in the mainstream of protein science, the use of the Bigelow scale to characterize caseins cannot be recommended; caseins cannot be fairly described as hydrophobic proteins and Pro does not behave as a hydrophobic residue.

In addition to the Letter to the Editor (Horne and Lucey, 2017), a fuller account of the objections to our work and conclusions was set out in a review article (Horne, 2017). In that publication, the original proposition that caseins are like giant soap molecules has been substantially modified but Horne continues to treat Pro as a hydrophobic residue. In place of hydrophobic tails, caseins are hypothesized to form small hydrophobic clusters among longer, mostly hydrophilic, sequences, corresponding to what we have called the P, Q-rich polar tracts. Horne uses a form of hydrophobic cluster analysis (Gaboriaud et al., 1987) developed for $\alpha$-helical regions in globular proteins and first applied to caseins by $\mathrm{Bu}$ et al. (2004). The hydrophobic residues in the hypothetical clusters are not adjacent in the sequence but are brought together only when the casein 
main chain forms $\alpha$-helical secondary structures. We consider the probability of such clusters to be low because although transient $\alpha$-helices are not unknown in IDPs, the $\alpha$-helical content of caseins is very low. This has been known from the earliest uses of optical rotatory dispersion to detect helical structures in proteins (Kresheck, 1965) and the low helical content was, in part, responsible for their reputation as "random coil" proteins. The lack of appreciable $\alpha$-helical structure has been confirmed by the latest technique of Raman optical activity (Syme et al., 2002). The advantage of Raman optical activity over circular dichroism and optical rotatory dispersion for the detection of secondary structure in IDPs is that the effect is more localized to the individual peptide bond and therefore sensitive to transient structures such as the predominant poly-Lproline helix in caseins. The constituent residues of the hypothetical clusters were identified by Horne using an unusually broad definition of hydrophobic residues to include the more polar Trp, Tyr, and Met side chains and further broadened to include Pro, a polar or hydrophilic residue and a well-known terminator of $\alpha$-helical structure. Horne also attempted to maximize the size of the hypothetical hydrophobic clusters. For example, in discussing a proposed cluster in $\alpha_{S_{2}}$-casein, Horne (2017) wrote that "between $\mathrm{Tyr}_{78}$ and $\mathrm{Val}_{113}$ several forced spatial turns are encountered at prolines in this extensive hydrophobic cluster. These proline residues should also be considered part of the hydrophobic clusters." In short, Horne has sought to maximize the possible number of residues involved in the hypothetical hydrophobic clusters by (1) including polar residues in his definition of hydrophobic residues, (2) proposing that the hydrophobic surface generated by one helix is continuous with those of neighboring helices even though they are separated by a helix-disrupting and relatively hydrophilic Pro residue, and (3) requiring that the $\alpha$-helical content of caseins be much greater than has been detected experimentally.

To summarize, we consider that the dual-binding model of the casein micelle was fundamentally flawed in its conception, drawing on an analogy with soap micelles and by supposing that the calcium phosphate nanoclusters exert a binding effect only by reducing casein net charge. We do not know the relative size of the free energy contributions that contribute to micelle formation but neither do Horne and Lucey. In consideration of the overall hydrophilic character of caseins, their unfolded conformation, lack of $\alpha$-helical structure, generally high prolyl residue content, and high solvation in their associated states, we think it is unreasonable to propose that hydrophobic interactions are dominant over all others. In effect, Horne has shoehorned caseins to fit the analogy with giant soap molecules by ignoring some and artificially combining and renaming other factors until they conform to his dual-binding model.

Horne and Lucey (Horne, 2017; Horne and Lucey, 2017) depart from accepted standards of scientific writing in several ways. First, they routinely misrepresent our findings and dissenting work from others in ways that favor their argument. In their Letter to the Editor, Horne and Lucey (2017) paraphrase our work 8 times, each time incorrectly. In his review, Horne (2017) paraphrases our work 22 times but only twice accurately. Three examples illustrate their practices. First, Horne and Lucey (2017) in the title of their letter, state that we dismissed hydrophobic interactions. We did not. What we dispute is the supposed dominance of the hydrophobic interactions. Second, Horne and Lucey (2017) state that De Kruif and Holt (2003) "tacitly accepted the notion of a dual-binding model (i.e., involving both attractive interactions and the formation of calcium phosphate nanoclusters as essential mechanisms for the assembly of casein micelles)." They did not, as demonstrated in the quotation from their review given above. Third, Horne (2017) states: "Holt and colleagues appear now to fully subscribe to the polymerizing dual-binding model of Horne (Horne, 1998)." We do not.

The second way that Horne and Lucey depart from accepted norms is in personalizing this scientific dispute by making derogatory remarks on the scientific competence of their opponents, questioning their ability to think clearly or explain their arguments in writing. Thus, in their Letter to the Editor, the final sentence of the opening paragraph and the second and third sentences of the final paragraph (Horne and Lucey, 2017) are unsupported denigrations. We have issued no diktats and airbrushed nothing; indeed, the people who act to suppress free thought and warn others to beware of our views are Horne and Lucey. By targeting us with immoderate language and multiple misrepresentations of our work, they may deter others from also expressing a contrary opinion. In both respects, Horne has proved to be a serial offender (Horne, 2006, 2017; Horne and Lucey, 2017). We look to reviewers and editors to control these excesses.

In concentrating on the issue of the relative importance of the hydrophobic effect, Horne and Lucey are drawing attention away from our most significant point. Whether the hydrophobic effect is important or not compared with other free energy changes involved in micelle formation is not the central issue. Our message in our reviews is that some IDPs provide better models for understanding caseins than soap molecules; casein researchers can call on a rich new source of ideas and methodologies to help them with their work. 


\section{REFERENCES}

Bigelow, C. C. 1967. On the average hydrophobicity of proteins and relation between it and protein structure. J. Theor. Biol. 16:187-211.

Bu, H.., S. M. Sood, and C. W. Slattery. 2004. The effect of C-terminal deletion on the folding and self-association of recombinant nonphosphorylated human beta-casein. Protein J. 23:509-517.

De Kruif, C. G., and C. Holt. 2003. Casein micelle structure, functions and interactions. Pages 233-276 in Advanced Dairy Chemistry. Vol. 1A: Proteins: Basic Aspects. 3rd ed. P. F. Fox and P. L. H McSweeney, ed. Kluwer, New York, NY.

Gaboriaud, C., V. Bissery, T. Benchetrit, and J. P. Mornon. 1987. Hydrophobic cluster analysis: An efficient new way to compare and analyse amino acid sequences. FEBS Lett. 224:149-155.

Holt, C., J. A. Carver, H. Ecroyd, and D. C. Thorn. 2013. Caseins and the casein micelle: their biological functions, structures and behaviour in foods. J. Dairy Sci. 96:6127-6146.

Horne, D. S. 1998. Casein interactions: Casting light on the black boxes, the structure in dairy products. Int. Dairy J. 8:171-177.

Horne, D. S. 2002. Casein structure, self-assembly and gelation. Curr. Opin. Colloid Interface Sci. 7:456-461.

Horne, D. S. 2006. Casein micelle structure: Models and muddles. Curr. Opin. Colloid Interface Sci. 11:148-153.

Horne, D. S. 2017. A balanced view of casein interactions. Curr Opin. Colloid Interface Sci. 28:74-86. https://doi.org/10.1016/j. cocis.2017.03.009.

Horne, D. S., and J. A. Lucey. 2017. Letter to the Editor: Hydrophobic interactions in the caseins: Challenging their dismissal by Holt et al. (2013). J. Dairy Sci. 100:5119-5120. https://doi.org/10.3168/ jds.2017-12668.

Janin, J. 1979. Surface and inside volumes in globular proteins. Nature 277:491-492
Kauzmann, W. 1959. Some factors in the interpretation of protein denaturation. Adv. Protein Chem. 14:1-63.

Kresheck, G. C. 1965. Conformation of casein in aqueous solution. Acta Chem. Scand. 19:375-382.

Kyte, J., and R. F. Doolittle. 1982. A simple method for displaying the hydropathic character of a protein. J. Mol. Biol. 157:105-132.

MacArthur, M. W., and J. M. Thornton. 1991. Influence of proline residues on protein conformation. J. Mol. Biol. 218:397-412.

Mao, A. H., S. L. Crick, A. Vitalis, C. L. Chicoine, and R. V. Pappu. 2010. Net charge per residue modulates conformational ensembles of intrinsically disordered proteins. Proc. Natl. Acad. Sci. USA 107:8183-8188.

Redwan, E. M., B. Xue, H. A. Almehdar, and V. N. Uversky. 2015. Disorder in milk proteins: Caseins, intrinsically disordered colloids. Curr. Protein Pept. Sci. 16:228-242.

Syme, C. D., E. W. Blanch, C. Holt, R. Jakes, M. Goedert, L. Hecht, and L. D. Barron. 2002. A Raman optical activity study of rheomorphism in caseins, synucleins and tau-New insight into the structure and behaviour of natively unfolded proteins. Eur. J. Biochem. 269:148-156.

Thorn, D. C., H. Ecroyd, J. A. Carver, and C. Holt. 2015. Casein structures in the context of unfolded proteins. Int. Dairy J. 46:2-11.

Uversky, V. N., J. R. Gillespie, and A. L. Fink. 2000. Why are "natively unfolded" proteins unstructured under physiologic conditions? Proteins 41:415-427.

van der Lee, R., M. Buljan, B. Lang, R. J. Weatheritt, G. W. Daughdrill, A. K. Dunker, M. Fuxreiter, J. Gough, J. Gsponer, D. T. Jones, P. M. Kim, R. W. Kriwacki, C. J. Oldfield, R. V. Pappu, P. Tompa, V. N. Uversky, P. E. Wright, and M. M. Babu. 2014. Classification of intrinsically disordered regions and proteins. Chem. Rev. 114:6589-6631. 